\title{
A Nationwide Multi-Center Questionnaire Survey on the Real-World State and Clinical Management of Poststroke Dementia in Japan
}

\author{
Kazuo Washida ${ }^{a, 1, *}$, Erika Kitajima ${ }^{a, b, 1}$, Tomotaka Tanaka ${ }^{a}$, Shuhei Ikeda ${ }^{a}$, Tetsuya Chiba ${ }^{a}$, \\ Kotaro Noda ${ }^{\mathrm{a}}$, Takeshi Yoshimoto ${ }^{\mathrm{a}}$, Kazuki Fukuma ${ }^{\mathrm{a}}$, Satoshi Saito ${ }^{\mathrm{a}}$ and Masafumi Ihara ${ }^{\mathrm{a}}$ \\ ${ }^{a}$ Department of Neurology, National Cerebral and Cardiovascular Center, Osaka, Japan \\ ${ }^{\mathrm{b}}$ Department of Medical Engineering, Faculty of Healthcare Sciences, Himeji Dokkyo University, Hyogo, Japan
}

Handling Associate Editor: Robert Friedland

Accepted 7 September 2021

Pre-press 4 October 2021

\begin{abstract}
.
Background: Poststroke dementia (PSD) is a serious problem for stroke survivors. However, there is still limited data on the real-world state and clinical management of PSD worldwide, and several countries already have a super-aged society.

Objective: We conducted a nationwide questionnaire survey to examine the real-world state and management of PSD in Japan.

Methods: A survey was conducted in the top 500 Japanese hospitals regarding the number of stroke patients treated between July 2018 and August 2019. Thirteen questions regarding PSD were mailed to doctors responsible for stroke management.

Results: Responses were obtained from 251 hospitals (50.2\%). The chief doctors responsible for stroke management answered the questionnaires. The median numbers of patients admitted annually with stroke in the departments of neurology and neurosurgery in the hospitals were 281.0 (interquartile range [IQR], 231.8-385.3) and 253.5 (IQR, 210.0-335.3), respectively, and most hospitals were acute care hospitals. Executive dysfunction was the most common cognitive dysfunction (10.9\%), followed by amnesia (9.5\%) and apathy (4.1\%). Surprisingly, many stroke survivors lived alone at home (23.7\%). Montreal Cognitive Assessment was significantly uncommon compared to Mini-Mental State Examination $(p<0.01)$. Furthermore, objective evaluation tests for behavioral and psychological symptoms of dementia were not often performed. Cognitive rehabilitation treatments were performed more often and earlier than drug treatments. The first drug of choice for PSD was predominantly donepezil (79.1\%), followed by galantamine (6.1\%), cilostazol (4.9\%), memantine (2.5\%), and rivastigmine $(1.8 \%)$.
\end{abstract}

Conclusion: Our study provides real-world evidence for the state of clinical practice related to PSD in Japan.

Keywords: Donepezil, executive dysfunction, living alone, Montreal Cognitive Assessment, poststroke dementia

\section{INTRODUCTION}

\footnotetext{
${ }^{1}$ These authors contributed equally to this work.

* Correspondence to: Kazuo Washida, MD, PhD, Department of Neurology, National Cerebral and Cardiovascular Center, 6-1 Kishibe-shimmachi, Suita, Osaka, 564-8565, Japan. Tel.: +81 6 6170 1070; Fax: +81 66170 1782; E-mail: washida@ncvc.go.jp.
}

Stroke has a huge public health burden and longterm medical care costs due to the high functional disability and mortality rate [1]. Stroke survivors could also have cognitive impairment and/or emotional problems in the chronic phase [2-4]; however, 
the effects of stroke on cognition and emotion have been underestimated for a long time.

Vascular cognitive impairment (VCI) or vascular dementia occurs as a result of brain ischemia and represents the second leading cause of dementia after Alzheimer's disease (AD) [5, 6]. There are several types of VCI: multi-infarct dementia, strategic single-infarct dementia, small vessel disease with dementia, hypoperfusive dementia, hemorrhagic dementia, and hereditary vasculopathy (e.g., cerebral autosomal dominant arteriopathy with subcortical infarcts and leukoencephalopathy, CADASIL) [7-9]. Among them, poststroke dementia (PSD) is predominantly defined as cognitive impairment occurring within 3 months after stroke [10].

PSD has become a major burden worldwide and is common in several countries, including Japan, that have already developed into a super-aged society. The world population is aging faster than ever. The United Nations reported that the percentage of population aged $\geq 65$ years in 2019 was $28.0 \%$ in Japan, $23.0 \%$ in Italy, $22.4 \%$ in Portugal, $22.1 \%$ in Finland, $21.9 \%$ in Greece, $21.6 \%$ in Germany, $21.3 \%$ in Bulgaria, 20.4\% in France, 20.2\% in Sweden, 18.5\% in the United Kingdom, $17.6 \%$ in Canada, $16.2 \%$ in the United States, $15.9 \%$ in Australia, and $15.1 \%$ in the Russian federation [11]. The prognosis of PSD is poor, even compared to that of AD [12]. Metaanalysis from several studies estimates that 1 in 10 patients suffer from dementia prior to the first stroke, 1 in 10 develop new dementia soon after the first stroke, and about one-third of stroke survivors suffer from PSD [13, 14]. The prevalence of cognitive impairment among patients with a history of stroke is similar to that of subjects 10 years older without a history of stroke [15]. Thus, early detection of stroke survivors with cognitive impairment is essential to improve their prognosis during the follow-up period [16].

The mortality rate of stroke has decreased due to recent advances in diagnostic imaging modalities, such as brain perfusion imaging as well as therapeutic advances, such as endovascular thrombectomy. Hence, the number of stroke survivors with complications, including cognitive impairment, dysphagia, and epilepsy, has been increasing [17]. Additionally, behavioral and psychological symptoms of dementia (BPSD), a difficult neuropsychiatric sequela, often afflicts stroke survivors and worsens their quality of life, becoming a major obstacle on the road to rehabilitation. Apathy, depression, irritation, anger, and delusions of persecution are well-known symptoms of BPSD. Poststroke apathy is a disturbance of motivation that is exhibited by low self-activation or emotional indifference. Poststroke depression is associated with worse functional outcomes, slower recovery, and poor quality of life [18]. Clinicians should be aware of the importance of PSD. We previously reported the real-world state of PSD from a global perspective, along with a systematic review of stroke and cognition in Africa [19] and the nationwide surveillance of poststroke complications in Japan [20]. However, there is still a marked lack of realworld information regarding the current state and management of PSD as well as limited evidence relating to their diagnosis and treatment; this is important as proper evaluation and management are essential for better prognoses in stroke survivors. Compared to $\mathrm{AD}$, healthcare professionals have less awareness of PSD. It is therefore hypothesized that the actual state and management of PSD patients remain unsatisfactory.

We conducted a questionnaire survey to examine the real-world state and management of PSD in Japan. This knowledge is necessary for a super-aged society like Japan in order to further develop and establish management strategies that meet the actual needs of stroke survivors with cognitive impairment, with the aim of extending their healthy life expectancy.

\section{MATERIALS AND METHODS}

\section{Subjects and methods}

A nationwide, cross-sectional, multi-center questionnaire survey was developed that targeted 500 facilities with the highest number of patients treated for stroke, based on the diagnosis procedure combination (DPC) disease name code published in the database of the Ministry of Health, Labor, and Welfare in Japan in 2017. The top 500 Japanese hospitals that treated the highest number of patients with stroke were selected in this survey. The method of outreach for this survey was physical mail. Responses to the questionnaires were requested from the chief doctors in charge of stroke care and management at the facilities. Fifty questions regarding poststroke complications (Supplementary Table 1), including dementia, dysphagia, epilepsy, pain, fall, depression, apathy, anxiety disorder, irritability, bladder-rectal disorder, and sleep disorder, were mailed to neurologists, neurosurgeons, and other experts responsible for the management of stroke. Out of a total of 50 questions, 13 questions regarding PSD were 
Table 1

Questionnaire items and responses

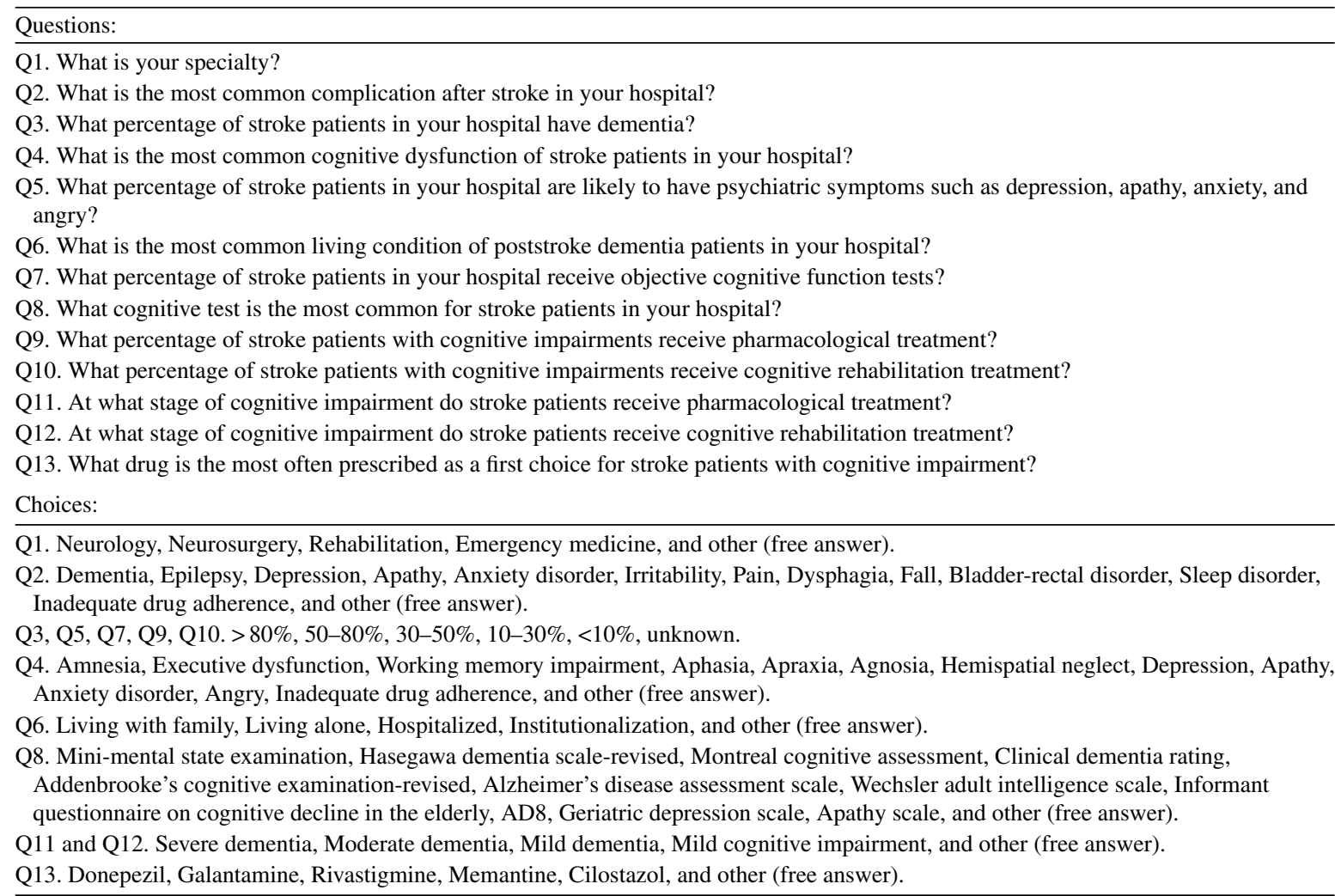

analyzed. The first questionnaire survey was conducted between July 2018 and August 2018, and a second survey request was made between January 2019 and February 2019 for facilities that did not provide responses to the first request.

As this study did not include patients' personal information and was a direct questionnaire survey for doctors, the ethics committee of our hospital identified this study as exempt from review and no deliberation was required by the committee. The data supporting the findings of this study are available from the corresponding author upon reasonable request.

\section{Survey contents}

The questionnaire items and responses are listed in Table 1. After confirming the department to which the respondents belonged, we requested that the respondents answer questions about PSD. The answer system was a check-box type one, and in the absence of appropriate options, a free answer box was provided.

\section{Statistical analysis}

Differences in responses to questions regarding the frequencies of cognitive function testing, drug treatment, and cognitive rehabilitation treatment, which were identified by the answers to Questions 8-12, were compared statistically and analyzed using Pearson's chi-square tests. JMP 14.0.0 software package (SAS Institute Inc., Cary, NC, USA) was used for all statistical analysis. A $p$-value of $<0.05$ was considered to represent a significant difference.

\section{RESULTS}

\section{Demographic data of the hospitals}

Responses were obtained from 251 out of 500 hospitals (response rate 50.2\%; 32.8\% after the first survey completion request was sent and $17.4 \%$ after the second survey completion request was sent). The chief doctor in charge of stroke care and management at each facility answered the questionnaires. Demographic data of the 251 hospitals that 
Table 2

Demographic data of the hospitals that participated in this study

\begin{tabular}{lccc}
\hline & $\begin{array}{c}\text { Neurology, } \\
n=144\end{array}$ & $\begin{array}{c}\text { Neurosurgery, } \\
n=95\end{array}$ & $\begin{array}{c}\text { Others, } \\
n=12\end{array}$ \\
\hline Type of hospital & & & \\
Primary (\%) & $0(0)$ & $0(0)$ & $0(0)$ \\
Secondary (\%) & $74(51.4)$ & $63(66.3)$ & $5(41.7)$ \\
Tertiary (\%) & $69(47.9)$ & $31(32.6)$ & $6(50.0)$ \\
Unknown (\%) & $1(0.7)$ & $1(1.1)$ & $1(8.3)$ \\
Region of hospital & & & \\
City (\%) & $140(97.2)$ & $93(97.9)$ & $11(91.7)$ \\
Province (\%) & $3(2.1)$ & $1(1.1)$ & $0(0)$ \\
Unknown (\%) & $1(0.7)$ & $1(1.1)$ & $1(8.3)$ \\
Number of hospital beds, median & 510.0 & 359.5 & 515.0 \\
(interquartile range) & $(400.0-702.0)$ & $(219.5-500.0)$ & $(315.0-670.0)$ \\
Number of stroke patients admitted & 281.0 & 253.5 & 260.0 \\
annually, median (interquartile range) & $(231.8-385.3)$ & $(210.0-335.3)$ & $(204.0-400.0)$ \\
Number of neurologist/neurosurgeon, & 5.0 & 5.0 & 5.0 \\
median (interquartile range) & $(3.0-9.0)$ & $(4.0-8.0)$ & $(3.0-6.0)$ \\
\hline
\end{tabular}

Data are presented as $\mathrm{n}(\%)$ or median (interquartile range). As for type and region of hospital, 3 hospitals (1 neurology, 1 neurosurgery, and 1 other department) were classified as unknown because responders only answered the department names but not the hospital names on the questionnaire. Others included 11 stroke and 1 rehabilitation departments.

answered the questionnaires are shown in Table 2. The median numbers of hospital beds in the departments of neurology and neurosurgery were 510.0 (interquartile range [IQR], 400.0-702.0) and 359.5 (IQR, 219.0-500.0), respectively. The median numbers of patients admitted annually with stroke in the departments of neurology and neurosurgery in the hospitals were 281.0 (IQR, 231.8-385.3) and 253.5 (IQR, 210.0-335.3), respectively. The median numbers of neurologist and neurosurgeons were 5.0 (IQR, 3.0-9.0) and 5.0 (IQR, 4.0-8.0), respectively. Hospitals were categorized as secondary $(n=142)$ or tertiary hospitals $(n=106)$, and most hospitals were acute care hospitals. Almost all hospitals were citybased hospitals $(n=244)$, with only a few provincial hospitals $(n=4)$. Three hospitals ( 1 neurology, 1 neurosurgery, and 1 other department) were classified as unknown for type and region because the responders only mentioned the department's name, not the hospital names, on the questionnaires. A total of 144 neurology departments (57.3\%), 95 neurosurgery departments (37.8\%), and 12 other departments (11 stroke and 1 rehabilitation) (4.8\%) responded to the questionnaires. (Q1; Tables 1 and 2).

\section{Characteristics of poststroke dementia}

In total, $30.9 \%$ of doctors answered that dementia was the most common complication in their hospitals, followed by dysphagia (29.3\%), apathy (16.3\%), bladder-rectal disorder $(6.5 \%)$, sleep disorder $(4.5 \%)$, falls (3.6\%), epilepsy (2.0\%), and others (6.9\%) (Q2; Fig. 1A).

The hospitals answered that the rate of stroke survivors with cognitive impairment was $>80 \%(0.8 \%)$, 50-80\% (6.5\%), 30-50\% (34.7\%), 10-30\% (39.5\%), $<10 \%(3.6 \%)$, and unknown (14.9\%) (Q3). The cognitive impairments of PSD included executive dysfunction (10.9\%), amnesia (9.5\%), and apathy (4.1\%) (Q4; Fig. 1A). The responses to Question 5 indicated that $10-30 \%$ of stroke survivors showed features of BPSD, such as apathy, depression, and anger (Q5).

Additionally, the living environment of patients with dementia after stroke also varied, with institutionalization $(50.7 \%)$ being the most common, followed by living alone (23.7\%) and living with family (20.9\%) (Q6; Fig. 1B).

\section{Cognitive tests for poststroke dementia}

As for cognitive testing after stroke, $54.9 \%$ of the hospitals performed objective cognitive tests for less than half of the patients, while $38.6 \%$ of the hospitals performed objective cognitive tests for more than half of patients; many of the hospitals did not routinely and objectively evaluate the level of cognition of stroke survivors using cognitive batteries (Q7; Fig. 2A).

The Mini-Mental State Examination (MMSE) (51.2\%) and revised Hasegawa Dementia Scale (HDS-R: Japanese local cognitive test) (44.9\%) were mainly used to assess the level of cognition of stroke 
A) Frequent poststroke complications and cognitive impairments observed after stroke

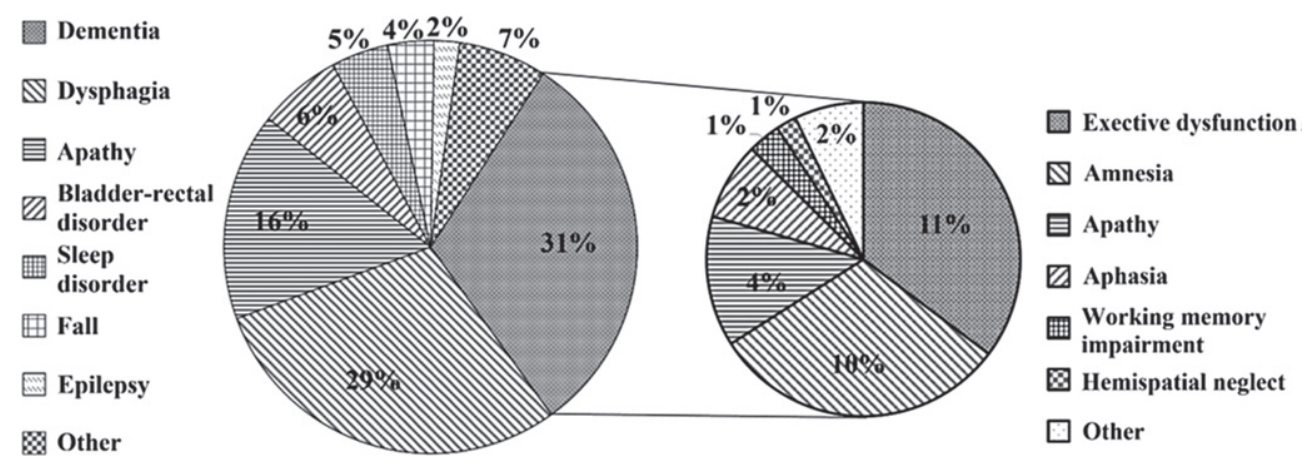

B) Living environment of patients with poststroke dementia

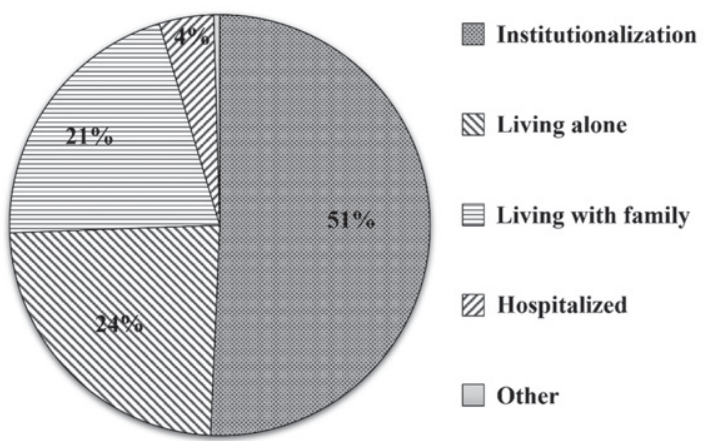

Fig. 1. Poststroke complications and living environment. A) In total, 30.9\% of doctors answered that dementia was the most common complication in their hospitals, followed by dysphagia (29.3\%), apathy (16.3\%), bladder-rectal disorder $(6.5 \%)$, sleep disorder (4.5\%), falls (3.6\%), epilepsy $(2.0 \%)$, and others $(6.9 \%)$. Considering subclassified cognitive dysfunction, $10.9 \%$ of doctors answered that executive dysfunction was the most common cognitive dysfunction in their hospitals, followed by amnesia (9.5\%) and apathy (4.1\%). B) Patients with dementia after stroke were most often institutionalized (50.7\%), living alone (23.7\%), or living with family (20.9\%).

survivors (Q8; Fig. 2B). However, Montreal Cognitive Assessment (MoCA), which is recommended for detecting PSD, [21] was significantly uncommonly used $(0.4 \%)$ compared to MMSE $(p<0.01)$. Furthermore, tests for BPSD, such as Geriatric Depression Scale (GDS) and apathy scale, were also extremely uncommon.

\section{Management of poststroke dementia}

From the management and therapeutic perspective, $16.9 \%$ of hospitals administered drug treatments for more than half of their patients, while $83.1 \%$ hospitals administered drug treatments for less than half of their patients (Q9; Fig. 3A). On the other hand, $42.7 \%$ of the hospitals undertook cognitive rehabilitation treatments for more than half of their patients and $43.1 \%$ of the hospitals performed cognitive rehabilitation treatments for less than half of their patients; the rate of cognitive rehabilitation treatment for more than half of the patients $(42.7 \%)$ was significantly higher than that of drug treatment $(16.9 \%)(p<0.01$, Q10; Fig. 3A).

As for the stage at which stroke survivors receive treatment, $39.1 \%$ of the hospitals administered drug therapy for patients with mild cognitive impairment (MCI) or mild dementia, while $51.1 \%$ of the hospitals administered drug therapy for patients with moderate or severe dementia. On the other hand, $60.2 \%$ of the respondents replied that they performed cognitive rehabilitation treatments for patients with MCI or mild dementia, while $28.6 \%$ stated that they performed drug treatments for patients with moderate or severe dementia; the rate of cognitive rehabilitation treatment for patients with MCI and mild dementia $(60.2 \%)$ was significantly higher than that of drug treatment $(39.1 \%)(p<0.01, \mathrm{Q} 11,12$; Fig. 3B). Cognitive rehabilitation treatments were undertaken at milder stages of dementia than drug treatments. 
A) Frequency of cognitive function testing

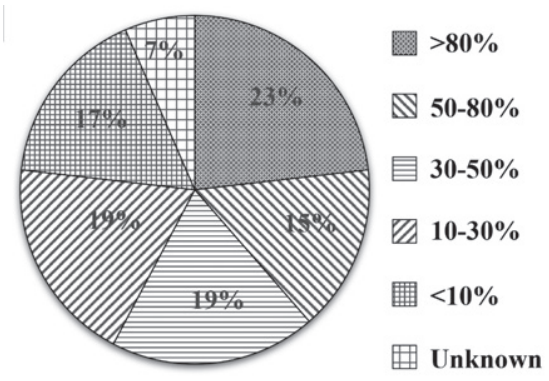

B) Type of cognitive function testing

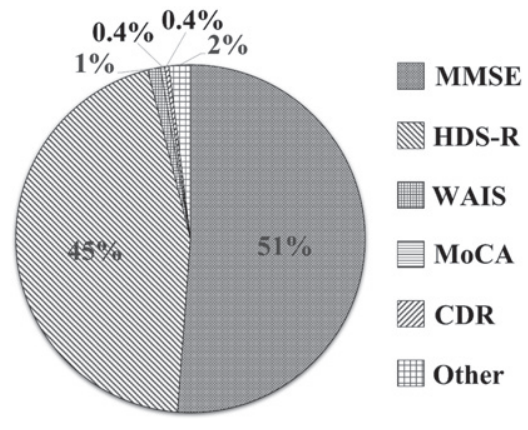

Fig. 2. Cognitive function testing. A) As regards cognitive function testing after stroke, the proportion of stroke survivors who were administered objective cognitive function tests was $>80 \%$ (23.2\%), 50-80\% (15.4\%), 30-50\% (19.1\%), 10-30\% (19.1\%), $<10 \%(16.7 \%)$, and unknown (6.5\%). B) MMSE (51.2\%), and HDS-R (44.9\%) were mainly used to assess the cognition of stroke survivors. However, MoCA was significantly uncommon $(0.4 \%)$ compared to MMSE $(p<0.01)$. MMSE, Mini-Mental State Examination; HDS-R, Hasegawa Dementia Scale-revised; MoCA, Montreal Cognitive Assessment; CDR, Clinical Dementia Rating; WAIS, Wechsler adult intelligence scale.

The most frequent first drug of choice used in the hospitals was predominantly donepezil $(79.1 \%)$, followed by galantamine (6.1\%), cilostazol (4.9\%), memantine $(2.4 \%)$, and rivastigmine $(1.8 \%)(\mathrm{Q} 13$; Fig. 4).

\section{DISCUSSION}

This nationwide multi-center questionnaire survey provides clarity regarding the real-world state and current medical managements of PSD from the perspective of healthcare professionals in Japan-a country characterized by a super-aged society. The most common cognitive impairments of PSD were executive dysfunction, followed by amnesia and apathy. Surprisingly, a quarter of stroke survivors were found to be living alone. MoCA was rarely used as a cognitive function test for stroke survivors. Cognitive rehabilitation was undertaken more often and at an earlier stage than drug treatment. The first drug of choice for dementia was predominantly donepezil, followed by galantamine, rivastigmine, and memantine. Based on these results, we discuss the current evidence and issues regarding PSD, a particularly problematic issue for stroke survivors in a super-aged society.

PSD often worsens quality of life and is an especially serious problem for stroke survivors. A 30-year nationwide population-based cohort study using data from Danish medical databases covering all Danish hospitals showed that the hazard ratio for dementia among stroke survivors was 1.80 after any stroke, 1.72 after ischemic stroke, and 2.70 after intracerebral hemorrhage, compared to that of the general population [22]. The socio-economic burden of PSD has been increasing because of the drastic increase of the number of stroke survivors in Japan and the Western world, where several countries already have super-aged societies [17]. However, there is still limited evidence about the proper diagnosis and treatment of PSD, and the current real-world clinical practice of PSD remains to be elucidated. Our nationwide survey revealed several real-world problems of stroke survivors in Japan, which has one of the most advanced aging societies in the world. Clinicians should exercise caution concerning the representative cognitive impairment after stroke revealed by this study, such as executive dysfunction, amnesia, and apathy. A national survey of Canadian occupational therapists' assessments of PSD also reported that the MMSE was often used incorrectly to evaluate the patient's cognition and that executive dysfunction (a critical component of poststroke assessment) was rarely assessed [23]. Furthermore, it should be emphasized that many stroke survivors live alone. Redfors et al. reported that 176 out of 600 poststroke patients (28.9\%) lived alone, which was associated with increased long-term mortality after ischemic stroke in Sweden [24]. This situation needs to be improved with medical as well as social support because living alone is also a strong risk factor for the progression of dementia [25].

Early detection and intervention should be executed because PSD predicts poorer survival in long-term follow-up [26]. In order to improve the prognosis of PSD, objective evaluation of cognitive function is first recommended [27]. The National Institute of Neurological Disorders and Stroke (NINDS) and the Canadian Stroke Network (CSN) emphasize cognitive and mood assessment for all stroke survivors, and it is increasingly recognized 
A) The rate of drug or cognitive rehabilitation treatments

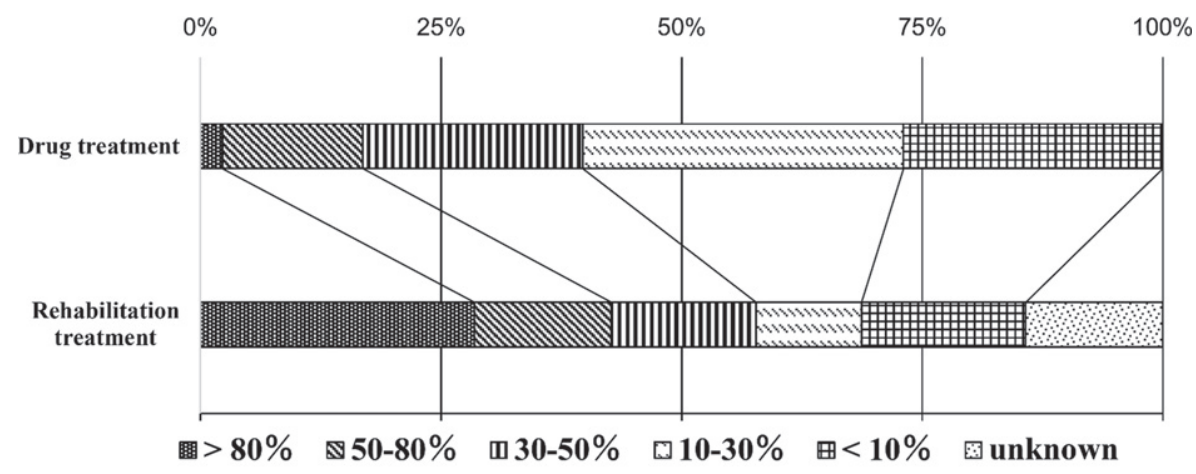

B) The stage at which stroke survivors receive drug or cognitive rehabilitation treatments

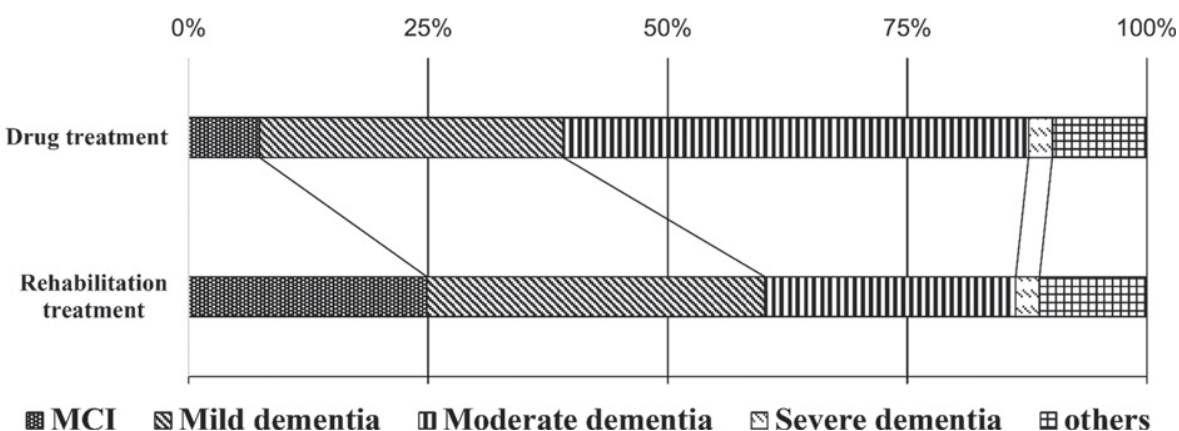

Fig. 3. Treatment of dementia after stroke. A) The rate of stroke survivors who received drug treatments was $>80 \%(2.3 \%), 50-80 \%(14.6 \%)$, $30-50 \%(22.8 \%), 10-30 \%(33.3 \%),<10 \%(27.0 \%)$, and unknown $(0 \%)$. The rate of stroke survivors who received cognitive rehabilitation treatments was $>80 \%$ (28.5\%), 50-80\% (14.2\%), 30-50\% (15.0\%), 10-30\% (11.0\%), <10\% (17.1\%), and unknown (14.2\%). Cognitive rehabilitation treatment was undertaken significantly more often and in more than half the patients (42.7\%) compared with drug treatment $(16.9 \%)(p<0.01)$. B) The stages of cognitive impairment at which stroke survivors who received drug treatments was mild cognitive impairment (MCI) $(7.4 \%)$, mild dementia (31.7\%), moderate dementia (48.5\%), severe dementia (2.5\%), and other $(9.9 \%)$. The stages of cognitive impairment at which stroke survivors who received cognitive rehabilitation treatments were MCI (24.9\%), mild dementia (35.3\%), moderate dementia $(26.1 \%)$, severe dementia $(2.5 \%)$, and other $(11.2 \%)$. The rate of cognitive rehabilitation for patients with MCI and mild dementia $(60.2 \%)$ was significantly higher than that of drug treatment $(39.1 \%)(p<0.01)$.

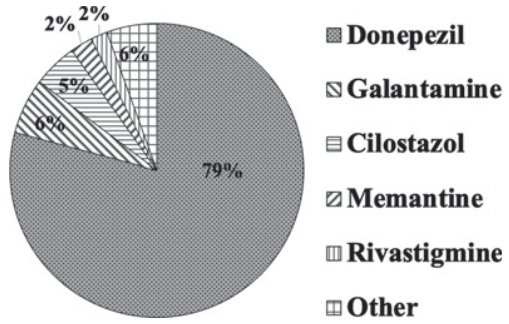

Fig. 4. Drugs used for poststroke dementia. The most frequent first drug of choice was predominantly donepezil $(79.1 \%)$, followed by galantamine $(6.1 \%)$, cilostazol $(4.9 \%)$, memantine $(2.4 \%)$, and rivastigmine $(1.8 \%)$.

that cognitive assessment should be part of the routine neurological examination in clinical practice [21, 28, 29]. However, our nationwide survey showed that many hospitals do not routinely and objectively evaluate cognition in stroke survivors using cognitive batteries. This fact implies that cumbersome examinations are usually avoided during routine assessment in many stroke hospitals in Japan. Additionally, among objective cognitive function tests, MMSE was commonly used, whereas MoCA, which is considered to have good sensitivity for PSD [30], was not. Our finding is in consistence with those of the other studies. A national survey among neurologists in the Netherlands reported that approximately half of the neurologists only sometimes, or even never, screened stroke survivors for cognitive and emotional problems [31]. A survey of Australian occupational therapists working with PSD also reported that MMSE were most frequently used, and most interventions were chosen based on their past experiences (88.3\%) and colleagues' opinions (77.4\%) [32]. MoCA is regarded to be suitable for evaluating PSD [33] and should be administered 
more actively to stroke survivors [34]. While MoCA is a time-consuming and laborious task for clinicians who are busy with daily clinical work, the NINDSCSN 5-minute protocol, which consists of only verbal tasks, could be recommended as a brief screening instrument for PSD [35, 36]. The etiology of PSD is complex and is related to the stroke itself as well as prestroke cognition; however, no hospital in this study used objective evaluation methods for prestroke cognition, such as Informant Questionnaire for Cognitive Decline in the Elderly (IQCODE). IQCODE is the most commonly employed assessment [29, $37,38]$. Patients with prestroke cognitive impairment usually have more severe ischemic stroke disabilities and worse prognosis $[39,40]$. Attention should also be paid to prestroke cognitive function in order to achieve a better prognosis in stroke survivors.

Our nationwide survey further demonstrated that BPSD was not objectively evaluated in detail using methods such as GDS and apathy scale in most hospitals. Stroke survivors often have emotional problems, such as apathy, depression, and anger. Stroke survivors with BPSD and depressive symptoms have a comparably higher risk of stroke and all-cause mortality [41]. Poststroke apathy acts as a barrier to meaningful participation in cognitive and physical rehabilitation [42]. BPSD is an important target of intervention in stroke survivors because BPSD can be ameliorated by medication and appropriate living conditions. For instance, early antidepressant administration for depression following stroke appears to enhance both physical and cognitive recovery from stroke and might increase survival by up to 10 years after stroke [43]. A guideline for healthcare professionals from the American Heart Association/American Stroke Association recommended the administration of a structured depression inventory such as the Patient Health Questionnaire-2 to routinely screen poststroke depression [44]. Therefore, clinicians should also be watchful for BPSD in stroke survivors as this is treatable and an important therapeutic target. It would be desirable to increase opportunities for objective evaluation of BPSD, which could prevent patients with stroke from developing cognitive impairment and emotional problems, resulting in better clinical outcome [45].

From the management and therapeutic perspective, there are two main treatment options for vascular dementia $(\mathrm{VaD})$ : drug and rehabilitation treatment [29]. In the current survey, the drug used as the first choice was predominantly donepezil, followed by galantamine, cilostazol, memantine, and rivastigmine. Donepezil was most often prescribed because it has been licensed for a long time, and it can be easily administered once daily. Additionally, generic versions of donepezil are already widely used in Japan, although it is controversial whether cholinesterase inhibitors should be used for PSD or not [46]. Acetylcholinesterase inhibitors, such as donepezil [47, 46], galantamine [48], and rivastigmine [49] were shown to improve VaD. Memantine, an N-methyl-D-aspartate (NMDA) receptor antagonist, could also be effective [50, 51]. However, acetylcholinesterase inhibitors and NMDA receptor antagonists are not currently approved for use in $\mathrm{VaD}$, including cases of PSD, and further clinical research is urgently needed to explore this therapeutic possibility.

Our survey revealed that cognitive rehabilitation treatment is more commonly undertaken by stroke survivors than drug treatment in daily clinical practice. Rehabilitation treatment is effective for preventing the progression of PSD [52]. A systematic review including nine studies demonstrated that increased physical activity improves cognitive function of stroke survivors [53]. A meta-analysis of randomized controlled trials also supported these findings [54]. Cognitive rehabilitation is also important for better prognosis [55]. Rehabilitation treatment is effective in mitigating $\mathrm{VaD}$ by increasing the bioavailability of neurotrophins and stimulating endothelial function [56]. Recently, it has become clear that multidomain interventions such as diet, exercise guidance, and cognitive function training can improve the level of cognition of stroke survivors $[57,58]$. Active, early treatment using medication and rehabilitation, as well as strict control of vascular risk factors such as hypertension and smoking could also significantly contribute to the reduction of cognitive impairment as proven by the Systolic Blood Pressure Intervention Trial (SPRNT-MIND) [59], the Cognitive Function and Ageing Study (CFAS) [60], and the Framingham studies [61].

The world is aging at a rapid rate and more than $20 \%$ of the population in 51 countries will be aged over 65 years by 2030 [11]. Many countries, such as Japan, Italy, Portugal, Finland, Greece, Germany, and Bulgaria, have already developed into a superaged society, and others, such as France, Sweden, Canada, the United Kingdom, and the United States, are in the pre-stage of a super-aged society [11]. Our survey clarified several important issues to consider regarding the state and management of PSD in Japan-a super-aged country. These issues could 


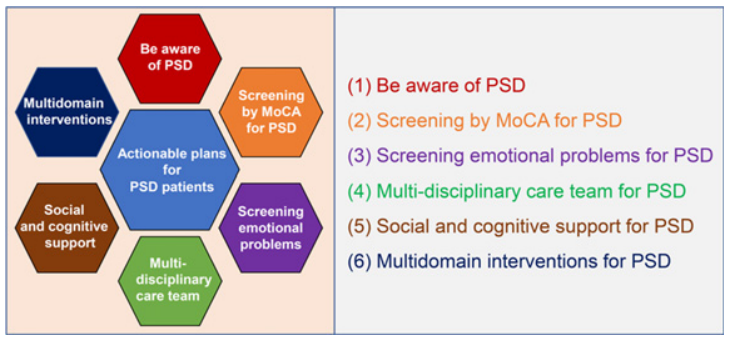

Fig. 5. Six actionable plans to improve prognosis and quality of life of poststroke dementia patients. (1) Be aware of PSD: Healthcare professionals should be aware of the importance of PSD and cautious about the representative cognitive impairment after stroke, such as executive dysfunction, amnesia, and apathy. (2) Screening by MoCA for PSD: MoCA or NINDS-CSN 5-minute protocol should be a part of the routine neurological examination for stroke survivors in daily clinical practice. (3) Screening emotional problems for PSD: Screening emotional problems, such as apathy, depression, and anger, should be a part of the routine neurological examination for stroke survivors in daily clinical practice. (4) Multi-disciplinary care team for PSD: Neuro-psychologists and cognitive/behavioral neurologists should be included in multidisciplinary care teams for PSD patients. (5) Social and cognitive support for PSD: Social and cognitive support for PSD patients that live alone should be executed. (6) Multidomain interventions for PSD: Multidomain interventions such as diet, exercise guidance, and cognitive training should be executed for PSD patients. PSD, poststroke dementia; MoCA, Montreal cognitive assessment; NINDS-CSN 5-minute protocol, The National Institute of Neurological Disorders and Stroke and the Canadian Stroke Network 5-minute protocol.

also be problematic in other super-aged and presuper-aged countries. Inappropriate and inadequate objective evaluation test for cognition and emotion for stroke survivors could cause high old age dependency ratio, which leads to high healthcare costs and long-term public health burden worldwide [27]. The increase in solitary stroke survivors could also worsen the prognoses [25] and it should not be ignored. However, the countermeasures for these problems remain unsatisfactory worldwide. Therefore, we propose six actionable plans for improving prognosis and quality of life of stroke survivors with cognitive impairment: 1) Be aware of PSD, 2) Screening by MoCA for PSD, 3) Screening emotional problems for PSD, 4) Multi-disciplinary care teams for PSD, 5) Social and cognitive support for PSD, and 6) Multidomain interventions for PSD (Fig. 5).

This study has several limitations. First, there is a possibility that the conditions of hospitals that manage more chronic stroke patients were not reflected in our results because the hospitals involved in this questionnaire survey mainly treat patients with acute stroke. Further surveys that reflect the situations in chronic care hospitals are needed in the future. Second, 249 responses were not obtained in this questionnaire survey (non-response rate $49.8 \%$ ), although we made the survey request twice. This non-response rate could have affected the results. Third, this study is based on information from the representative directors of the hospital. The chief doctors in charge of stroke care and management at each facility answered the questionnaires, as accurately as possible. However, there is a possibility that some doctors might have answered based on a rough percentage estimate as per their perceptions, although these possible biases could be mitigated by the responses of experienced chief doctors responsible for stroke management. Actual conditions and medical management of PSD based on the strict hospital statistics or population-based data are necessary to confirm the validity of our findings and their generalizability in future studies. Fourth, the top 500 hospitals that treated the highest number of stroke patients were selected in this study because we tried to investigate the real-world state and management of PSD at representative hospitals that provide standard stroke treatment in Japan. However, possible selection bias could have affected the study results. Further studies with random sampling that minimize selection bias and reflect the more generalized state and management of PSD are needed in future. Fifth, this survey was conducted in Japan and the findings do not necessarily reflect the actual state of other countries in the world. However, several countries, such as Italy, Portugal, and Finland, have also developed into a super-aged society and countries such as France, Sweden, and the United Kingdom, are in the pre-stage of a super-aged society. Hence, our findings can be a useful reference worldwide to further develop and establish management strategies that meet the actual needs of stroke survivors with cognitive impairment, with the aim of extending their healthy life expectancy.

\section{CONCLUSION}

In this study, we clarified the real-world state and management regarding PSD in Japan. Clinicians should be aware of the importance of PSD, although data in this regard remain unsatisfactory. Clinical evidence regarding PSD is scarce in Japan and across the world, and detailed and clear guidelines are imperative. The information in this study could be useful for early diagnosis and treatment of stroke survivors, thus improving their prognosis and quality of life. 


\section{ACKNOWLEDGMENTS}

We are sincerely indebted to all the responsible doctors who answered the questions. We are also indebted to Ms. Sasaki, Ms. Ohashi, and Ms. Wada for their excellent secretarial assistances.

This work was supported by a Grant-in-Aid for Scientific Research on Priority Areas from the Japanese Ministry of Education, Science and Culture: 19K21321 (to K.W.). This work was also supported by a Grant-in-Aid from Osakagasgroup Welfare Foundation (to K.W.). This research was also funded by Japan Agency for Medical Research and Development (AMED), grant number 21ek0109516h0001.

Authors' disclosures available online (https:// www.j-alz.com/manuscript-disclosures/21-5006r1).

\section{SUPPLEMENTARY MATERIAL}

The supplementary material is available in the electronic version of this article: https://dx.doi.org/ 10.3233/JAD215006.

\section{REFERENCES}

[1] Benjamin EJ, Blaha MJ, Chiuve SE, Cushman M, Das SR, Deo R, de Ferranti SD, Floyd J, Fornage M, Gillespie C, Isasi CR, Jiménez MC, Jordan LC, Judd SE, Lackland D, Lichtman JH, Lisabeth L, Liu S, Longenecker CT, Mackey RH, Matsushita K, Mozaffarian D, Mussolino ME, Nasir K, Neumar RW, Palaniappan L, Pandey DK, Thiagarajan RR, Reeves MJ, Ritchey M, Rodriguez CJ, Roth GA, Rosamond WD, Sasson C, Towfighi A, Tsao CW, Turner MB, Virani SS, Voeks JH, Willey JZ, Wilkins JT, Wu JH, Alger HM, Wong SS, Muntner P; American Heart Association Statistics Committee and Stroke Statistics Subcommittee (2017) Heart disease and stroke statistics-2017 update: A report from the American Heart Association. Circulation 135, e146-e603.

[2] Nakling AE, Aarsland D, Naess H, Wollschlaeger D, Fladby T, Hofstad H, Wehling E (2017) Cognitive deficits in chronic stroke patients: Neuropsychological assessment, depression, and self-reports. Dement Geriatr Cogn Dis Extra 29, 283-296.

[3] Douven E, Köhler S, Schievink SHJ, van Oostenbrugge RJ, Staals J, Verhey FRJ, Aalten P (2018) Baseline vascular cognitive impairment predicts the course of apathetic symptoms after stroke: The CASPER Study. Am J Geriatr Psychiatry 26, 291-300

[4] Arwert HJ, Meesters JJL, Boiten J, Balk F, Wolterbeek R, Vliet Vlieland TPM (2018) Poststroke depression: A longterm problem for stroke survivors. Am J Phys Med Rehabil 97, 565-571.

[5] O'Brien JT, Thomas A (2015) Vascular dementia. Lancet 386, 1698-1706.

[6] Matsui Y, Tanizaki Y, Arima H, Yonemoto K, Doi Y, Ninomiya T, Sasaki K, Iida M, Iwaki T, Kanba S, Kiyohara $Y$ (2009) Incidence and survival of dementia in a general population of Japanese elderly: The Hisayama study. J Neurol Neurosurg Psychiatry 80, 366-370.

[7] Román GC, Tatemichi TK, Erkinjuntti T, Cummings JL, Masdeu JC, Garcia JH, Amaducci L, Orgogozo JM, Brun A, Hofman A, et al. (1993) Vascular dementia: Diagnostic criteria for research studies. Report of the NINDS-AIREN International Workshop. Neurology 43, 250-260.

[8] Jiwa NS, Garrard P, Hainsworth AH (2010) Experimental models of vascular dementia and vascular cognitive impairment: A systematic review. J Neurochem 115, 814-828.

[9] Gooch J, Wilcock DM (2016) Animal models of vascular cognitive impairment and dementia (VCID). Cell Mol Neurobiol 36, 233-239.

[10] Jacquin A, Binquet C, Rouaud O, Graule-Petot A, Daubail B, Osseby GV, Bonithon-Kopp C, Giroud M, Béjot Y (2014) Post-stroke cognitive impairment: High prevalence and determining factors in a cohort of mild. stroke. J Alzheimres Dis 40, 1029-1038.

[11] United Nations, Department of Economic and Social Affairs, population Division (2019). World Population Ageing 2019: Highlights (ST/ESA/SER.A/430).

[12] Carod-Artal FJ, Egido JA (2009) Quality of life after stroke: The importance of a good recovery. Cerebrovasc Dis $\mathbf{2 7}$ Suppl 1, 204-214.

[13] Pendlebury ST (2009) Stroke-related dementia: Rates, risk factors and implications for future research. Maturitas 64, 165-171.

[14] Pendlebury ST, Rothwell PM (2009) Prevalence, incidence, and factors associated with pre-stroke and post-stroke dementia: A systematic review and meta-analysis. Lancet Neurol 8, 1006-1018.

[15] De Ronchi D, Palmer K, Pioggiosi P, Atti AR, Berardi D, Ferrari B, Dalmonte E, Fratiglioni L (2007) The combined effect of age, education, and stroke on dementia and cognitive impairment no dementia in the elderly. Dement Geriatr Cogn Disord 24, 266-273.

[16] Gorelick PB, Scuteri A, Black SE, Decarli C, Greenberg SM, Iadecola C, Launer LJ, Laurent S, Lopez OL, Nyenhuis D, Petersen RC, Schneider JA, Tzourio C, Arnett DK, Bennett DA, Chui HC, Higashida RT, Lindquist R, Nilsson PM, Roman GC, Sellke FW, Seshadri S (2011) Vascular contributions to cognitive impairment and dementia: A statement for healthcare professionals from the American Heart Association/American Stroke Association. Stroke 42, 2672-2713.

[17] Kalaria RN, Akinyemi R, Ihara M (2016) Stroke injury, cognitive impairment and vascular dementia. Biochim Biophys Acta 1862, 915-925.

[18] Arauz A, Rodríguez-Agudelo Y, Sosa AL, Chávez M, Paz F, González M, Coral J, Díaz-Olavarrieta C, Román GC (2014) Vascular cognitive disorders and depression after first-ever stroke: The Fogarty-Mexico Stroke Cohort. Cerebrovasc Dis 38, 283-289.

[19] Akinyemi RO, Owolabi MO, Ihara M, Damasceno A, Ogunniyi A, Dotchin C, Paddick SM, Ogeng'o J, Walker R, Kalaria RN (2019) Stroke, cerebrovascular diseases and vascular cognitive impairment in africa. Brain Res Bull 145, 97-108.

[20] Ikeda S, Washida K, Tanaka T, Kitajima E, Chiba T, Fukuma K, Yoshimoto T, Saito S, Hattori Y, Ihara M (2021) A nationwide multi-center questionnaire survey on the realworld state and issues regarding post-stroke complications in japan. J Stroke Cerebrovasc Dis 30, 105656.

[21] Eskes GA, Lanctot KL, Herrmann N, Lindsay P, Bayley M, Bouvier L, Dawson D, Egi S, Gilchrist E, Green T, Gubitz G, 
Hopper T, Khan A, King A, Kirton A, Moorhouse M, Smith EE, Green J, Foley N, Salter K, Swartz RH (2015) Canadian stroke best practice recommendations: Mood, cognition and fatigue following stroke practice guidelines, update 2015 . Int J Stroke 10, 1130-1140.

[22] Corraini P, Henderson VW, Ording AG, Pedersen L, Horvath-Puho E, Sorensen HT (2017) Long-term risk of dementia among survivors of ischemic or hemorrhagic stroke. Stroke 48, 180-186.

[23] Korner-Bitensky N, Barrett-Bernstein S, Bibas G, Poulin V (2011) National survey of Canadian occupational therapists' assessment and treatment of cognitive impairment post-stroke. Aust Occup Ther J 58, 241-250.

[24] Redfors P, Isaksén D, Lappas G, Blomstrand C, Rosengren A, Jood K, Jern C (2016) Living alone predicts mortality in patients with ischemic stroke before 70 years of age: A long-term prospective follow-up study. BMC Neurol 16, 80.

[25] Grande G, Vetrano DL, Cova I, Pomati S, Mattavelli D, Maggiore L,Cucumo V, Ghiretti R, Vanacore N, Mariani C, Rizzuto D (2018) Living alone and dementia incidence: A clinical-based study in people with mild cognitive impairment. J Geriatr Psychiatry Neurol 31, 107-113.

[26] Melkas S, Oksala NK, Jokinen H, Pohjasvaara T, Vataja R, Oksala A, Kaste M, Karhunen PJ, Erkinjuntti T (2009) Poststroke dementia predicts poor survival in longterm follow-up: Influence of prestroke cognitive decline and previous stroke. J Neurol Neurosurg Psychiatry 80, 865-870.

[27] Brainin M, Tuomilehto J, Heiss WD, Bornstein NM, Bath PM, Teuschl Y, Richard E, Guekht A, Quinn T (2015) Poststroke cognitive decline: An update and perspectives for clinical research. Eur J Neurol 22, 229-238, e213-226.

[28] Hachinski V, Iadecola C, Petersen RC, Breteler MM, Nyenhuis DL, Black SE, Powers WJ, DeCarli C, Merino JG, Kalaria RN, Vinters HV, Holtzman DM, Rosenberg GA, Wallin A, Dichgans M, Marler JR, Leblanc GG (2006) National Institute of Neurological Disorders and StrokeCanadian Stroke Network vascular cognitive impairment harmonization standards. Stroke 37, 2220-2241.

[29] Mijajlovic MD, Pavlovic A, Brainin M, Heiss WD, Quinn TJ, Ihle-Hansen HB, Hermann DM, Assayag EB, Richard E, Thiel A, Kliper E, Shin Y, Kim Y, Choi S, Jung S, Lee Y, Sinanović O, Levine DA, Schlesinger I, Mead G, Milošević V, Leys D, Hagberg G, Ursin MH, Teuschl Y, Prokopenko S, Mozheyko E, Bezdenezhnykh A, Matz K, Aleksić V, Muresanu D, Korczyn AD, Bornstein NM (2017) Post-stroke dementia - a comprehensive review. BMC Med 15, 11.

[30] Nakaoku Y, Oishi N, Hase Y, Hase M, Saito S, Mitsueda T, Matsui M, Toyoda K,Nagatsuka K, Kalaria RN, FukuyamaH, Ihara M, Takahashi R (2018) Montreal cognitive assessment score correlates with regional cerebral blood flow in post-stroke patients. Clin Neurol Neurosurg 174, 68-74.

[31] Slenders J, Van den Berg-Vos R, Visser-Meily J, van Heugten C, Kwa V (2021) Screening and follow-up care for cognitive and emotional problems after transient ischaemic attack and ischaemic stroke: A national, cross-sectional, online survey among neurologists in the Netherlands. BMJ Open 11, e046316.

[32] Koh CL, Hoffmann T, Bennett S, McKenna K (2009) Management of patients with cognitive impairment after stroke: A survey of Australian occupational therapists. Aust Occup Ther 56, 324-331.

[33] Stolwyk RJ, O’Neill MH, McKay AJ, Wong DK (2014) Are cognitive screening tools sensitive and specific enough for use after stroke? A systematic literature review. Stroke 45, 3129-3134.

[34] Pendlebury ST, Cuthbertson FC, Welch SJ, Mehta Z, Rothwell PM (2010) Underestimation of cognitive impairment by mini-mental state examination versus the Montreal Cognitive Assessment in patients with transient ischemic attack and stroke: A population-based study. Stroke 41, 12901293.

[35] Kennedy RE, Wadley VG, McClure LA, Letter AJ, Unverzagt FW, Crowe M, Nyenhius D, Kelley BJ, Kana B, Marceaux J, Tamura MK, Howard V, Howard G (2014) Performance of the NINDS-CSN 5-minute protocol in a national population-based sample. J Int Neuropsychol Soc 20, 856-867.

[36] Lim JS, Oh MS, Lee JH, Jung S, Kim C, Jang MU, Lee SH, Kim YJ, Kim Y, Park J, Kang Y, Yu KH, Lee BC (2017) Prediction of post-stroke dementia using ninds-csn 5-minute neuropsychology protocol in acute stroke. Int Psychogeriatr 29, 777-784.

[37] Harrison JK, Fearon P, Noel-Storr AH, McShane R, Stott DJ, Quinn TJ (2015) INFORMANT QUESTIONNAIRE on Cognitive Decline in the Elderly (IQCODE) for the diagnosis of dementia within a secondary care setting. Cochrane Database Syst Rev 10, CD010772.

[38] Tang WK, Chan SS, Chiu HF, Wong KS, Kwok TC, Mok V, Ungvari GS (2003) Can iqcode detect poststroke dementia? Int J Geriatr Psychiatry 18, 706-710.

[39] Bejot Y, Duloquin G, Crespy V, Durier J, Garnier L, Graber M, Giroud M (2020) Influence of preexisting cognitive impairment on clinical severity of ischemic stroke: The Dijon Stroke Registry. Stroke 51, 1667-1673.

[40] Zheng F, Yan L, Zhong B, Yang Z, Xie W (2019) Progression of cognitive decline before and after incident stroke. Neurology 93, e20-e28.

[41] Eurelings LS, van Dalen JW, Ter Riet G, Moll van Charante EP, Richard E, van Gool WA, Almeida OP, Alexandre TS, Baune BT, Bickel H, Cacciatore F, Cooper C, de Craen TA, Degryse JM, Di Bari M, Duarte YA, Feng L, Ferrara N, Flicker L, Gallucci M, Guaita A, Harrison SL, Katz MJ, Lebrão ML, Leung J, Lipton RB, Mengoni M, Ng TP, Østbye T, Panza F, Polito L, Sander D, Solfrizzi V, Syddall HE, van der Mast RC, Vaes B, Woo J, Yaffe K; ICARA Study Group (2018) Apathy and depressive symptoms in older people and incident myocardial infarction, stroke, and mortality: A systematic review and meta-analysis of individual participant data. Clin Epidemiol 10, 363-379.

[42] Mayo NE, Fellows LK, Scott SC, Cameron J, WoodDauphinee S (2009) A longitudinal view of apathy and its impact after stroke. Stroke 40, 3299-3307.

[43] Robinson RG, Jorge RE (2016) Post-stroke depression: A review. Am J Psychiatry 173, 221-231.

[44] Winstein CJ, Stein J, Arena R, Bates B, Cherney LR, Cramer SC, Deruyter F, Eng JJ, Fisher B, Harvey RL, Lang CE, MacKay-Lyons M, Ottenbacher KJ, Pugh S, Reeves MJ, Richards LG, Stiers W, Zorowitz RD; American Heart Association Stroke Council, Council on Cardiovascular and Stroke Nursing, Council on Clinical Cardiology, and Council on Quality of Care and Outcomes Research (2016) Guidelines for adult stroke rehabilitation and recovery: A guideline for healthcare professionals from the American Heart Association/American Stroke Association. Stroke 47, e98-e169.

[45] Ihle-Hansen H, Thommessen B, Fagerland MW, Oksengard AR, Wyller TB, Engedal K, Fure B (2014) Effect on anxiety and depression of a multifactorial risk factor intervention 
program after stroke and TIA: A randomized controlled trial. Aging Ment Health 18, 540-546.

[46] Chang WH, Park YH, Ohn SH, Park CH, Lee PK, Kim YH(2011) Neural correlates of donepezil-induced cognitive improvement in patients with right hemisphere stroke: A pilot study. Neuropsychol Rehabil 21, 502-514.

[47] Malouf R, Birks J (2004) Donepezil for vascular cognitive impairment. Cochrane Database Syst Rev 1, CD004395.

[48] Auchus AP, Brashear HR, Salloway S, Korczyn AD, De Deyn PP, Gassmann-Mayer C (2007) Galantamine treatment of vascular dementia: A randomized trial. Neurology 69, 448-458.

[49] Birks J, McGuinness B, Craig D (2013) Rivastigmine for vascular cognitive impairment. Cochrane Database Syst Rev, CD004744.

[50] Orgogozo JM, Rigaud AS, Stoffler A, Mobius HJ, Forette F (2002) Efficacy and safety of memantine in patients with mild to moderate vascular dementia: A randomized, placebo-controlled trial (MMM 300). Stroke 33, 1834-1839.

[51] Wilcock G, Mobius HJ, Stoffler A, MMM 500 group (2002) A double-blind, placebo-controlled multicentre study of memantine in mild to moderate vascular dementia (mmm500). Int Clin Psychopharmacol 17, 297-305.

[52] Longley V, Peters S, Swarbrick C, Bowen A (2019) What factors affect clinical decision-making about access to stroke rehabilitation? A systematic review. Clin Rehabil 33, 304-316.

[53] Cumming TB, Tyedin K, Churilov L, Morris ME, Bernhardt J (2012) The effect of physical activity on cognitive function after stroke: A systematic review. Int Psychogeriatr 24, 557-567.

[54] Oberlin LE, Waiwood AM, Cumming TB, Marsland AL, Bernhardt J, Erickson KI (2017) Effects of physical activity on poststroke cognitive function: A meta-analysis of randomized controlled trials. Stroke 48, 3093-3100.

[55] Nair RD, Lincoln NB (2007) Cognitive rehabilitation for memory deficits following stroke. Cochrane Database Syst Rev 18, CD002293.

[56] Dao E, Hsiung GR, Liu-Ambrose T (2018) The role of exercise in mitigating subcortical ischemic vascular cognitive impairment. J Neurochem 144, 582-594.
[57] Ngandu T, Lehtisalo J, Solomon A, Levälahti E, Ahtiluoto S, Antikainen R, Bäckman L, Hänninen T, Jula A, Laatikainen T, Lindström J, Mangialasche F, Paajanen T, Pajala S, Peltonen M, Rauramaa R, Stigsdotter-Neely A, Strandberg T, Tuomilehto J, Soininen H, Kivipelto M (2015) A 2 year multidomain intervention of diet, exercise, cognitive training, and vascular risk monitoring versus control to prevent cognitive decline in at-risk elderly people (finger): A randomised controlled trial. Lancet 385, 2255-2263.

[58] Teuschl Y, Ihle-Hansen H, Matz K, Dachenhausen A, Ratajczak P, Tuomilehto J, Ursin MH, Hagberg G, Thommessen $\mathrm{B}$, Øksengård AR (2018) Multidomain intervention for the prevention of cognitive decline after stroke - a pooled patient-level data analysis. Eur J Neurol 25, 1182-1188.

[59] SPRINT MIND Investigators for the SPRINT Research Group, Williamson JD, Pajewski NM, Auchus AP, Bryan RN, Chelune G, Cheung AK, Cleveland ML, Coker LH, Crowe MG, Cushman WC, Cutler JA, Davatzikos C, Desiderio L, Erus G, Fine LJ, Gaussoin SA, Harris D, Hsieh MK, Johnson KC, Kimmel PL, Tamura MK, Launer LJ, Lerner AJ, Lewis CE, Martindale-Adams J, Moy CS, Nasrallah IM, Nichols LO, Oparil S, Ogrocki PK, Rahman M, Rapp SR, Reboussin DM, Rocco MV, Sachs BC, Sink KM, Still CH, Supiano MA, Snyder JK, Wadley VG, Walker J, Weiner DE, Whelton PK, Wilson VM, Woolard N, Wright JT Jr, Wright CB (2019) Effect of intensive vs standard blood pressure control on probable dementia: A randomized clinical trial. JAMA 321, 553-561.

[60] Matthews FE, Arthur A, Barnes LE, Bond J, Jagger C, Robinson L, Brayne C (2013) A two-decade comparison of prevalence of dementia in individuals aged 65 years and older from three geographical areas of England: Results of the Cognitive Function and Ageing Study I and II. Lancet 382, 1405-1412.

[61] Satizabal CL, Beiser AS, Chouraki V, Chêne G, Dufouil C, Seshadri S (2016) Incidence of dementia over three decades in the Framingham Heart Study. NEngl J Med 374, 523-532. 